\title{
Entendimento dos Controles e Possíveis Conflitos de Privacidade nas Redes Sociais Online
}

\author{
Talita S. Costa ${ }^{1,2}$, Marbilia P. Sergio ${ }^{1}$, Regina Marin ${ }^{2}$ \\ Centro de Tecnologia da Informação Renato Archer (CTI) \\ Caixa Postal 13069-901 - Campinas - SP - Brazil \\ 'Instituto Federal de Educação, Ciência e Tecnologia de São Paulo (IFSP-Campinas) \\ Caixa Postal 13069-901 - Campinas - SP - Brazil \\ \{tscosta;marbilia.sergio\}@cti.gov.br, rpmmarin@gmail.com
}

\begin{abstract}
The Online Social Networks (OSNs) provide settings for the user to define his privacy profile. In publishing with other authors, OSNs prioritize the publisher's posting rules, generating privacy conflicts. The objective of this work is to analyze the user's level of understanding about the privacy configuration and perception regarding the controls and possible existence of privacy conflict in OSNs, especially when occurs the usage of TAG. Through a Survey methodology it was found that privacy settings are still inefficient and there is a need to implement strategies in the OSNs to avoid conflicts.
\end{abstract}

Resumo. As Redes Sociais Online (RSOs) disponibilizam configurações para que o usuário defina seu perfil de privacidade. Na publicação com outros autores, as RSOs priorizam a regra de postagem do editor podendo gerar conflitos de privacidade. O objetivo deste trabalho é analisar o nível de entendimento do usuário sobre a configuração de privacidade e a percepção quanto aos controles e possivel existência de conflito de privacidade nas RSOs, em especial quando ocorre o uso de TAG. Através da metodologia Survey obteve-se que as configurações de privacidade ainda são ineficientes $e$ que há necessidade de se implementar uma estratégia nas RSOs para evitar conflitos.

\section{Introdução}

As Redes Sociais Online (RSOs) são os websites mais visitados do mundo [Nielsen 2012]. As RSOs permitem que se reproduza na Internet o comportamento da sociedade, interações e relacionamentos. Cria-se perfis e se estabelece vínculos com outras pessoas. Nestes ambientes, [Marin, Piolle and Bidan 2014] afirmam que as atividades mais populares no ambiente de RSO é a marcação e criação de TAGs e que o compartilhamento, entre pessoas que aparecem numa mesma foto e referenciadas por $T A G$. Como vários usuários conectados podem ter diferentes preocupações com a privacidade em relação aos dados a serem compartilhados, conflitos de privacidade ocorrem podendo aumentar o risco potencial de vazar informações confidenciais $[\mathrm{Hu}$ 2011].

As RSOs existentes solucionam conflitos ao priorizar o dono da informação, e acabam desfavorecendo os demais envolvidos na mesma. $\mathrm{Na}$ literatura, foram encontradas propostas para equacionar conflitos em forma de estratégia. A estratégia "Negar" foi proposta por [AmirH, HamidR and Frank 2007]. A aplicação desta estratégia no contexto das RSOs, sempre prevaleceria o negar se pelo menos um dos usuários negar a publicação da imagem. Já, a estratégia de [Yamada, Kim and Perrig 
2012] privilegia a decisão da "Maioria" sobre controle de acesso. Assim, esta estratégia adaptada ao mesmo cenário de compartilhamento, é resolvido pela vontade da maioria.

Geralmente, os controles implementados nas RSOs deveriam refletir o que é declarado nas suas políticas de privacidade, o que de fato não é observado [Anthonysamy 2011]. Uma vez colocado a divergência entre a política esperada e os controles implementados nas RSOs, analisar a percepção do usuário em relação aos controles de privacidade existentes passa a ser necessário para que medidas cabíveis possam ser tomadas na busca de um ambiente mais seguro.

Esta pesquisa utilizou a metodologia Survey através de um questionário que visou obter informações quanto a percepção do usuário e capacidade de compreensão dos controles de privacidades existentes no Facebook, e se o mesmo está preparado para usá-los, para gerenciar possíveis conflitos existente nestes ambientes focando em discutir o entendimento de privacidade no que se refere ao uso de TAGs em imagens fotográficas. Este artigo está organizado em três seções. A Seção 1 apresenta um breve conceito de privacidade e conflito em Redes Sociais Online, a Seção 2 apresenta os resultados observados após análise das respostas obtidas, e finalmente a Seção 3 coloca as considerações finais.

\section{Levantamento da percepção dos usuários sobre os controles de privacidade e conflitos}

Utilizando a metodologia Survey, foi realizada uma pesquisa por questionário online (https://docs.google.com/uc?export=download\&id=16L5PYZvmPObTC9L3fCRXmGDfzfaweDll). O questionário foi respondido por 129 pessoas, porém utilizados somente as 99 respostas das pessoas que responderam todo o questionário. O período da coleta de dados foi de outubro de 2018 até fevereiro de 2019.

\subsection{Quanto aos controles de privacidade nas RSOs}

A Figura 1 ilustra que apesar de apenas $53.5 \%$ dos participantes afirmarem entende o efeito da aplicação dos controles de privacidade, $65.7 \%$ afirmam saber usar na sua timeline e 73,7\% afirmam saber usa-los em fotos o que deixa em aberto se de fato comprendem o efeito do uso desses controles. No entanto, a convergência mais próxima entre as respostas pode ser observada entre as afirmação de 53,5\% que entende os controles e os 49,5\% que afirmam saber usar os controles com a "TAG".

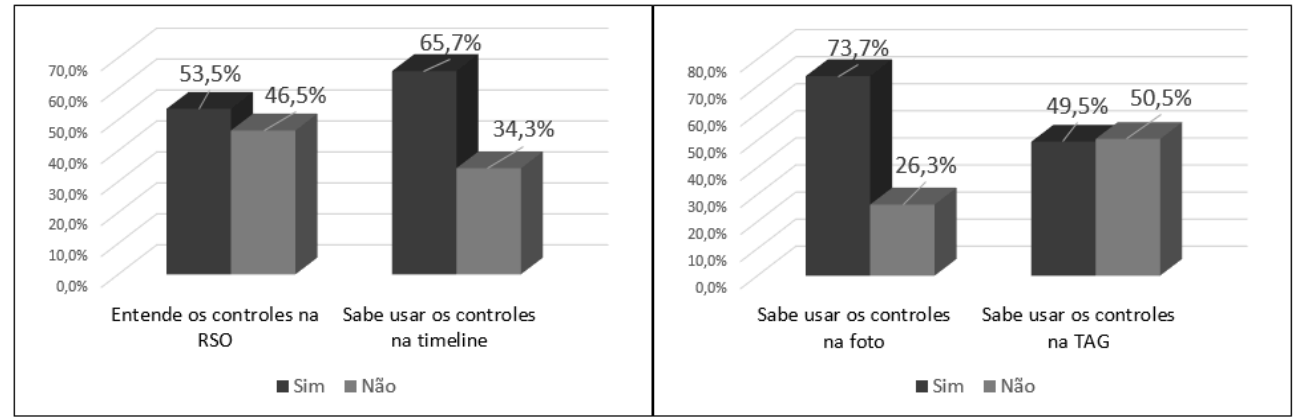

Figura 1 Utilidades dos controles de privacidade nas RSOs. 
Quanto a ter lido a política de privacidade, a Figura 2 mostra que 74,7\% dos participantes não leram e logo não sabem sobre seu conteúdo. No entanto, também 63.6\% não acredita estar protegido só pelo fato de existir a política de privacidade. Comparando este resultado com as pesquisas realizadas por [Zainab and Mamuna 2012] e [Williams, Agarwal and Wigand 2015] em relação ao gerenciamento de privacidade no Facebook observou-se que não houve significativa mudança neste cenário. [Zainab and Mamuna 2012] obteve que $87.9 \%$ das pessoas que não leram as políticas de privacidades enquanto [Williams, Agarwal and Wigand 2015] obteve a porcentagem de $70 \%$.

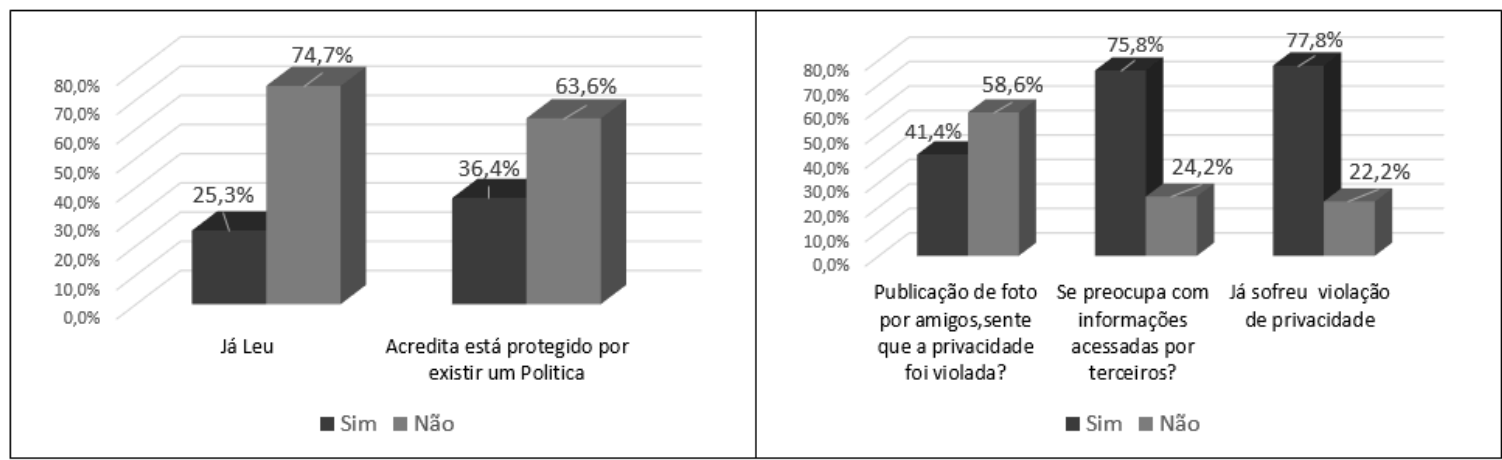

Figura 2 Políticas e violação de privacidade.

Quanto a violação da privacidade, 58.6\% dos participantes respondeu que não sente que sua privacidade foi violada quando um amigo publica uma foto sua. Nota-se que a maioria, $77.8 \%$ dos participantes nunca sofreu violação de privacidade, e $75.8 \%$ dos participantes dizem estar preocupados com o fato de suas informações serem acessadas por terceiros.

\subsection{Quanto ao grau de entendimento sobre conflitos de privacidade com uso de TAG.}

Na Figura 3 tem-se que quando apresentado a um autor a situação hipotética de uma consulta prévia aos coautores sobre a publicação de uma foto, qual seria a sua decisão se: somente um negasse ou se a maioria negasse. Obteve-se $89.9 \%$ afirmando que não postariam a foto se somente um envolvido não desejasse, no entanto, $90.9 \%$ não publicaria a foto caso a maioria dos coautores não desejasse. Os resultados demonstram que os usuários estão conscientes do efeito de uma postagem indesejada. Assim, se fosse adotada as estratégias de "Negar" e da "Maioria", a margem de conflitos de privacidade seria de $10 \%$ das situações.

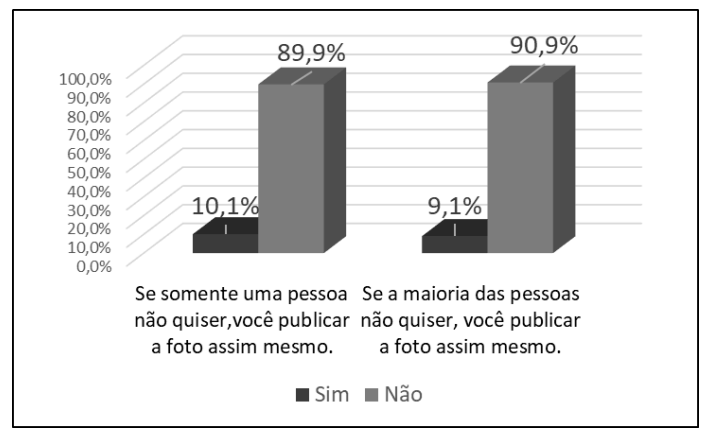

Figura 3 Conflitos de privacidade das estratégias negar e maioria. 


\section{Considerações Finais}

$\mathrm{Na}$ análise qualitativa sobre as percepções dos usuários proposta neste trabalho sobre conflitos de privacidade, obteve-se que os usuários apesar de não lerem as polícias de privacidade existentes, afirmam estar preocupados com a privacidade de suas informações. Notou-se que apesar de não compreenderem, eles usam os controles de privacidade disponibilizados pelas RSOs. Assim como, a grande maioria dos usuários afirma respeitar a vontade dos coautores das imagens. Se as RSOs concedessem aos autores e coautores das fotos a possibilidade de interagir sobre os recursos e preferências, na maioria dos casos não existiriam conflitos. Especialmente no uso de TAG, a maioria dos usuários afirma respeitar as estratégias de negação, nestes casos, o conflito se seria reduzido a somente $10 \%$ das situações.

\section{Agradecimentos}

Agradecemos ao CTI, IFSP e ao CNPq.

\section{Referências}

AmirH, C., HamidR C. and FrankWm, T. (2007) A unified conflict resolution algorithm. In Secure Data Management, volume 4721 of Lecture Notes in Computer Science, pages 1-17. Springer Berlin Heidelberg.

Anthonysamy, P., Rashid, A. and Greenwood, P. (2011) Do the privacy policies reflect the privacy controls on social networks? In: SocialCom/PASSAT. [S.1.]: IEEE, p. $1155-1158$.

Besmer, A. and Richter Lipford, H. (2010) Moving beyond untagging: photo privacy in a tagged world. In: Proceedings of the SIGCHI Conference on Human Factors in Computing Systems. ACM. p. 1563-1572.

Hu, H., Ahn, G. and Jorgensen, J. (2011) Detecting and resolving privacy conflicts for collaborative data sharing in online social networks. In: Proceedings of the 27th Annual Computer Security Applications Conference. ACM.

Marin, R., Piolle, G. and Bidan, C. (2014) Equity-preserving management of privacy conflicts in social network systems. In: The Sixth ASE International Conference on Privacy, Security, Risk and Trust (PASSAT), Cambridge, MA, USA.

Nielsen. H. (2012) Reports and Insights: The Social Media Report . Disponível em: $<$ http://www.nielsen.com/us/en/insights/reports/2012/state-of-the-media-the-socialmedia-report-2012>. Acesso em: 9 setembro. 2018.

Williams, T. L., Agarwal, N. and Wigand, R. T. (2014) Protecting Private Information: Current Attitudes Concerning Privacy Policies. The Sixth ASE International Conference on Privacy, Security, Risk and Trust (PASSAT), Cambridge, MA, USA.

Zainab, A. and Mamuna, K. (2012) Users' Perceptions on Facebook's Privacy Policies. ARPN Journal of Systems and Software, v. 2, n. 3, p 119-125.

Yamada, A., Kim, T. H. and Perrig, A. (2012) Exploiting privacy policy conflicts in online social networks. CMU-CyLab-12-005. 